Bio-grafia Escritos sobre la Biologia y su Enseñanza.

Edición Extra-Ordinaria. ISSN 2027-1034 P.p 930 - 938

Memorias del VII Encuentro Nacional de Experiencias en la Enseñanza de la Biología y la Educación Ambiental y II Congreso Nacional de Investigación en la Enseñanza de la Biología

\title{
ANÁLISIS Y REFLEXIONES AL REALIZAR PASANTÍAS DOCTORALES EN EDUCACIÓN, INTERNACIONAL Y NACIONAL
}

\section{ANALYSIS AND REFLECTIONS ON DOCTORAL INTERNSHIPS IN EDUCATION, INTERNATIONAL AND NATIONAL}

\section{Robinson Roa Acosta ${ }^{1}$}

\section{Resumen}

En este documento se recogen las reflexiones y análisis que se han desarrollado, por parte de quien realiza este escrito, a propósito de dos pasantías doctorales realizadas, una en la Universidad de Buenos Aires y la otra en la Universidad el Valle. En este sentido, se presentan elementos que surgen de la experiencia con los grupos de investigación en el contexto de sus culturas, constituciones teóricas, epistemológicas y metodológicas, al igual que los alcances que tiene la pasantía para la tesis, el desarrollo de la formación como investigador y las conexiones que se fortalecen entre los grupos de investigación.

Palabras Clave: Pasantía doctoral, educación, investigación, tesis.

\section{Abstract}

This document contains the reflections and analyzes that have been developed, by the person who made this paper, purposely made two doctoral internships, one at the University of Buenos Aires and the other in the University of Valle. In this sense, are presented elements that are based on experience with research groups in the context of their cultures, constitutions theoretical, epistemological and methodological, as well as having the internship scope for the thesis, the development of training as researcher and strengthen the connections between the research groups.

Key words: Internship doctoral, education, research, thesis

\footnotetext{
'Estudiante del Doctorado Interinstitucional en Educación, sede Universidad Pedagógica Nacional. Profesor Investigador Departamento de Biología y Departamento de Postgrados. Universidad Pedagógica Nacional.
} 
Bio-grafia Escritos sobre la Biologia y su Enseñanza.

Edición Extra-Ordinaria. ISSN 2027-1034 P.p 930 - 938

Memorias del VII Encuentro Nacional de Experiencias en la Enseñanza de la

Biología y la Educación Ambiental y II Congreso Nacional de Investigación en la Enseñanza de La Biología

\title{
Introducción
}

Como uno de los requisitos en la formación en el Doctorado Interinstitucional en Educación - DIE (Universidad Francisco José se Calda; Universidad del Valle; Universidad Pedagógica Nacional) se encuentra la realización de pasantías nacionales y/o internacionales, las cuales

\begin{abstract}
“...son espacios de formación doctoral, en las que los estudiantes deben realizar una serie de actividades académicas que contribuyan a la realización de la tesis y por tanto forman parte integral de las actividades académicas de su plan de estudios... los estudiantes pueden realizar una o varias pasantías en universidades vinculadas académicamente a los grupos de investigación del programa, orientada(s) a la consecución de bibliografía o materiales de difícil acceso, la aproximación a los objetos de estudio propios del grupo que recibe al estudiante mediante la realización de actividades académicas propias del grupo, la aplicación de determinadas metodologías desarrolladas por el mismo, por citar algunos ejemplos." (DIE)
\end{abstract}

En este sentido, de común acuerdo con el director de la tesis "Configuración del conocimiento didáctico profesional del profesor de ciencias para la enseñanza de la biotecnología en Colombia. Propuesta de investigación" (Roa, 2012), se llevó a cabo el respectivo contacto entre el grupo de investigación Conocimiento Profesional del Profesor de Ciencias del Departamento de Biología de la Universidad Pedagógica Nacional y los grupos Didáctica de la Biología -Centro de Formación e Investigación en Enseñanza de las Ciencias (CeFIEC)- y Ciencia, Acciones y Creencias -Instituto de Pedagogía y Educación-, los cuales corresponden respectivamente, a la Universidad de Buenos Aires y Universidad del Valle, con el fin de acordar mutualmente un plan de pasantía según la instancia institucional y los intereses del tutor de la pasantía, director de la tesis y pasante. En términos generales, en las pasantías se tuvo como actividades fundamentales: asesorías del tutor, participación en cursos doctorales, revisión y sistematización bibliográfica, participación y asistencia en eventos -encuentro de estudiantes de doctorado, paneles, seminarios-, visitas a librerías y ferias de libro, reuniones con los grupos de investigación, elaboración preliminar de escritos para publicar, entre otros.

Acorde a la intensión con la suscrita ponencia se presentaran a continuación algunos análisis y reflexiones que tienen que ver con lo que se adquiere para la formación como investigador y lo que aporta en la realización de la tesis, al igual 
Bio-grafia Escritos sobre la Biologia y su Enseñanza.

Edición Extra-Ordinaria. ISSN 2027-1034 P.p 930 - 938

Memorias del VII Encuentro Nacional de Experiencias en la Enseñanza de la Biología y la Educación Ambiental y II Congreso Nacional de Investigación en la Enseñanza de La Biología

que para la conexión entre los grupos de investigación, se presentan algunas conclusiones. Por el espacio destinado para este escrito no es posible describir cada una de las actividades.

\section{La pasantía y la formación para la investigación}

Es indudable que las representaciones que los ciudadanos y los investigadores realizan sobre los objetos, contextos, fenómenos, problemas -mundo inmediato o lejano, a decir, empírico y real, respectivamente- están directamente relacionados con el mundo que frecuentan, en este, el ambiente cultural impregna de sentidos y significados la vida de las personas. La lengua, las formas de comunicación, y con ellas los valores, tradiciones y artefactos -dispositivos- se convierten en estructuras generadas desde las mismas a su vez que generadoras reproductoras- de las mismas. Pero la lengua y el lenguaje per se no son vivos estos tiene sentido en tanto que los que tienen vida se la dan.

Precisamente, Foucault (2010) escribía que:

"Los códigos fundamentales de una cultura -los que rigen su lenguaje, sus esquemas perceptivos, sus cambios, sus técnicas, sus valores, la jerarquía de sus prácticas- fijan de antemano para cada hombre los ordenes empíricos con los cuales tendrá algo que ver y dentro de los cuales se reconocerá. En el otro extremo del pensamiento, las teorías científicas o las interpretaciones de los filósofos explican por qué existe un orden en general, a qué ley general obedece, qué principio puede dar cuenta de él, por qué razón se establece este orden y no aquel otro. (p. 13-14).

Las personas por sí mismas acuden a permanecer dentro de la cultura que los hace ser lo que son, es esta la que configura modelos de pensar, de ver el mundo, de sentir, pero a decir de Nietzsche en su libro "Ecce Homo. Cómo se llega a ser lo que se es" no solo se trata de la cultura sino además de otros aspectos -tales como el clima, alimentación- que aún cuando pueden parecer irrisorios para constitución y producción de conocimiento tienen fuertes connotaciones con lo que se es. Al referente este filósofo vehementemente escribía:

"...El tempo [ritmo] del metabolismo mantiene los pies del espíritu; el "espíritu" mismo, en efecto, no es más que una especie de ese metabolismo. ... en qué 
Bio-grafia Escritos sobre la Biologia y su Enseñanza.

Edición Extra-Ordinaria. ISSN 2027-1034 P.p 930 - 938

Memorias del VII Encuentro Nacional de Experiencias en la Enseñanza de la

Biología y la Educación Ambiental y II Congreso Nacional de Investigación en la Enseñanza de La Biología

lugares hay y ha habido hombres ricos en espíritu... donde el genio tuvo su hogar de manera casi necesaria: todos ellos poseen un aire magníficamente seco. París, la Provenza, Florencia, Jerusalén, Atenas...estos nombres demuestran una cosa: el genio está condicionado por el aires seco, por el cielo puro, es decir, por un metabolismo rápido, por la posibilidad de recobrar una y otra vez cantidades grandes, incluso gigantescas, de fuerza." (p. 37)

La ignorancia in physiologicis [en cuestiones de fisiología] -el maldito "idealismo"- es la auténtica fatalidad en mi vida, lo superfluo y estúpido en ella, algo de lo que no salió nada bueno y para lo cual no hay ninguna compensación, ningún descuento. Por la consecuencias de este "idealismo" me explico yo todos los desaciertos, todas las grande aberraciones del instinto y todas las "modestias" que me han apartado de la tarea de mi vida, así por ejemplo, el haberme hecho filólogo..." (p. 38)

Así pues, aquello que hace ser lo que se es no es únicamente causa de las tradiciones, creencias, costumbres, valores, sino que las características geográficas generan estados particulares que influyen en el ingenio; conocer el funcionamiento de la cultura en el sentido anterior conduce a generar conciencia de lo que se es en la cultura y lo que es la cultura para la formación del ser. Es precisamente en razón de ello que Nietzsche, a partir de y en la catarsis que realizada, elabora el híbrido fisiología-idealismo como constituyentes fundamentales de su explicación sobre cómo se es lo que se es, adicional a esto, el autor describe en varios de los apartados del libro la función que cumplen los libros y la educación -en perspectiva cultural-, aun cuando para esta última sea mejor poner de presente su libro "Sobre el porvenir de nuestras escuelas".

La distancia entre las culturas hace que esta adquiera propiedades y entendimientos que pueden ser compartidos en algunos aspectos al igual que disentidos justamente por la dinámica específica que se ha generado en cada una. Ahora, si se piensa en la cultura académica, científica, de investigadores, quizá lo que podría haber de distinto en la cultura general, en la cual están inmersos, es que el investigador busca, o debería hacerlo, construir conocimiento que le permita explicar, razonar, describir, interpretar problemas que tienen vinculo con la realidad en diferentes niveles, desde los más próximos a realidad inmediata hasta los más alejados de la realidad -no obstante, que de allí proceden en principio-. Esto está diciendo que el investigador, en la idea de entender en mundo, entra en el uso de relaciones entre los conceptos que le ayudan a representar sus interés, preocupaciones respecto a algo que lo inquieta, los niveles de relaciones que 
Bio-grafia Escritos sobre la Biologia y su Enseñanza.

Edición Extra-Ordinaria. ISSN 2027-1034 P.p 930 - 938

Memorias del VII Encuentro Nacional de Experiencias en la Enseñanza de la Biología y la Educación Ambiental y II Congreso Nacional de Investigación en la Enseñanza de La Biología

estable entre los conceptos, a propósito de lo que investiga, hace que se distancie de la realidad, no empero que esta está siempre presente de varias maneras como ya se buscó superfluamente mostrar.

En este sentido, es latente que existe la constante de que la teoría o modelos respondan a la realidad desde la cual surgieron, es decir, que el investigador debe estar alerta de que la teoría tenga una función, podría decirse que utilidad, para la realidad que en si no es científica aun cuando puede tener función no para una cultura sino para todas las culturas en tanto el razonamiento corresponde e implica en extensión una generalidad para explicar tópicos que se expresan en todas. Esto pone a pensar respecto al origen del conocimiento construido y el alcance que tiene para la cultura a la que llega.

Esto puesto en el terreno específico de la investigación en didáctica, pedagogía o educación, nos lleva a pensar si la discusión recurrente respecto a la función de la teoría y la práctica en la acción profesional del profesor se encuentran conjugadas o responden a realidades distintas. Precisamente dar sentido a esta dicotomía pone en entredicho la validez-confiabilidad del conocimiento que construyen los investigadores.

En el campo de investigación de las ciencias sociales y humanas tener en cuenta dichos aspectos es motivo de múltiples discusiones por cuanto que los resultados de las investigaciones no tienen como medio y fin la reproducción de los resultados sino más bien interpretar para comprender y así mejorar - de ser posible- la práctica. Desde luego que en esto tiene cabida clara en la formación de los profesionales de la enseñanza, ya que los referentes teóricos de los futuros profesores no constriñen a la teoría y la práctica por separado, sino que todo lo contrario tiene fuerte connotación con la acción profesional.

Así pues, la pasantía tiene un fuerte contenido de aprendizaje en tanto se pueden ver y entender las dinámicas que los grupos de investigación tienen -del que provengo y al que fui-. Los discursos y las estructuras que se sostienen tienen directa relación con la cultura académica que se vive como resultado de los autores que se leen y analizan buscando dar interpretación y explicación a sus objetos de investigación. En esto, se encuentra cómo las teorías son usadas para conformar miradas particulares sobre los estudios. Con lo cual la mirada rígida y 
Bio-grafia Escritos sobre la Biologia y su Enseñanza.

Edición Extra-Ordinaria. ISSN 2027-1034 P.p 930 - 938

Memorias del VII Encuentro Nacional de Experiencias en la Enseñanza de la Biología y la Educación Ambiental y II Congreso Nacional de Investigación en la Enseñanza de La Biología

doctrinaria tiende a desvanecerse dentro de epistemologías más flexibles en las que las coexistencias de las mismas se hace cada vez más latentes.

\section{La Pasantía Y La Tesis De Doctorado}

Las pasantías resultaron muy nutritiva, atinadas ya que al compartir con los grupos de investigación -investigadores, tesistas, profesores, amigos, muchos de ellos conjugados- se pueden aprender no sólo asuntos relacionados con la construcción del conocimiento, tradiciones, metodologías, cuerpos teóricos, sino también su calidad humana; el compartir diferentes espacios -bar (cafetería, restaurante), análisis, reflexión, caminatas, librerías, bibliotecas, etc.,- ayuda a comprender los valores que hay en quienes practican la investigación, en este sentido, si que tiene valor incalculable la sociología de la ciencia ya que poder transmitir esto a las personas puede ayudar a acercarlos al conocimiento no sólo en sus modos de producción sino también de las cualidades de quienes investigan.

Luego entonces, la pasantía aporta para la tesis en la idea de hacer una aproximación a lo que puede implicar la sociología de la ciencia para la enseñanza, al igual que de las posturas epistemológicas, ontológicas, ideológicas y filosóficas que orientan su acción en la investigación y en la enseñanza. La participación en las diferentes actividades permitió elaborar, reforzar, recordar, tener presente y dar peso a la tesis en tópicos que constituyen el conocimiento del profesor de ciencias, en particular de Biología.

Vivenciar la práctica de la enseñanza en el aula de clase en la formación de profesores, en la cual se pone en escena la metodología y los contenidos a enseñar resulta una experiencia muy enriquecedora, sobre todo por la experiencia y calidad de los profesores investigadores que la precedían.

Así también, el acceso a la bibliografía elaborada dentro de esta cultura -sus artículos y libros-, la participación en las clases, de las reuniones del grupo de investigación, foros, seminarios, análisis conjuntos con estudiante de doctorado, aportan de manera significativa al posicionamiento epistemológico y metodológico en el trabajo de investigación que se adelanta.

Igualmente, la bibliografía consultada y seleccionada en cuanto a la educación en biotecnología, la biotecnología, y el conocimiento del profesor constituyen 
Bio-grafia Escritos sobre la Biologia y su Enseñanza.

Edición Extra-Ordinaria. ISSN 2027-1034 P.p 930 - 938

Memorias del VII Encuentro Nacional de Experiencias en la Enseñanza de la Biología y la Educación Ambiental y II Congreso Nacional de Investigación en la Enseñanza de La Biología

documentos clave para el desarrollo de la tesis que se adelanta. El poder argumentar la perspectiva que sigue la tesis y las perspectivas en los trabajos de tesis de doctorado genera rupturas y posturas muchas veces dogmaticas en la metodología y cuerpos teóricos de conocimiento preestablecidos y los que se pueden construir.

La organización del currículo en la formación de profesores de Biología profesorado en Argentina- de la Universidad de Buenos Aires y el programa que se desarrolla en argentina "Por qué biotecnología" amplia las perspectivas desde donde pueden ser enseñada la biotecnología en los países de acuerdo a las estructuras políticas, económicas, científicas y tecnológicas.

En el grupo de investigación de la Universidad del Valle se pudo encontrar una dinámica particular para compartir y realizar actividades académicas y de investigación en la cual el fervor de sus participantes por el análisis y discusión apasionada eran, al igual que en el CeFIEC, la constante imperiosa, esencial para poder expresar el pensar y sentir respecto a la didáctica, profesor, educación, enseñanza, aprendizaje, currículo, epistemología, entre muchas otras cosas.

\section{A manera de Conclusión}

Las pasantías realizadas constituyen una riqueza para la formación como investigador, permiten encontrarse con otras culturas, en todo el sentido de la palabra, que posibilitan entender por qué se es como se es, a la vez que facilitan romper, confirmar, compartir ordenes teóricos y metodológicos.

Poder compartir gramáticas, pensamientos, razonamientos propios de los grupos de investigación, ayuda a fortalecer lazos de amistad y de investigación, en la cual todos estamos pensando en la intensión de explicar en fenómeno de la enseñanza y lo ontológico, y epistemológico, en la formación profesional de los profesores de Biología.

La tesis recoge y se fortalece de los desarrollos en investigación de los dos grupos de investigación tanto en lo relacionado con el conocimiento del profesor como en la educación en enseñanza de la biotecnología, saber que esta ha sido punto de discusión en los dos grupos, en diferentes instancias (grupo de investigación e 
Bio-grafía Escritos sobre la Biología y su Enseñanza.

Edición Extra-Ordinaria. ISSN 2027-1034 P.p 930 - 938

Memorias del VII Encuentro Nacional de Experiencias en la Enseñanza de la Biología y la Educación Ambiental y II Congreso Nacional de Investigación en la Enseñanza de la Biología

institucional en la Universidad del Valle, y especialmente a nivel de nacional en la Argentina) permite pensar en las posibilidades de la enseñanza, educación y formación de profesores de Bilogía para la enseñanza de la biotecnología en Colombia.

\section{Agradecimientos}

Mis más sinceros agradecimientos a los tutores de las pasantías: Doctor Leonardo Gonzáles Galli (Universidad de Buenos Aires), Doctor Alfonso Claret Zambrano (Universidad del Valle), a los integrantes de sus grupos de investigación Didáctica de la Biología y Ciencia, Acciones y Creencias, respectivamente, por su recibimiento y calidad humana. Al doctor Edgar Orlay Valbuena Ussa, director de la tesis y del grupo de investigación Conocimiento Profesional del Profesor de Ciencias, por su apoyo en este proceso y en muchos otros, por su amistad.

\section{Referencias}

Doctorado Interinstitucional en Educación. Reglamentación procedimiento para realización de pasantías. En: tias.pdf

http://www.pedagogica.edu.co/admin/docs/1339108851 procedimientopasan

Foucault, M. (2010). Las palabras y las cosas. Una arqueología de las ciencias sociales. México: Siglo veintiuno editores.

Nietzsche, F. (2005). Ecce Homo. Cómo se llega a ser lo que se es. México: Grupo Editorial Tomo, S.A. de C.V.

Nietzsche, F. (2009). Sobre el porvenir de nuestras escuelas. Barcelona: Fabula Tusquests Editores.

Roa, R. (2012). Configuración del conocimiento didáctico profesional del profesor de ciencias para la enseñanza de la biotecnología en Colombia.

Propuesta de investigación. Memorias: III Congreso Nacional de Investigación en Educación en Ciencias y Tecnología (EDUCYT). II Congreso Iberoamericano de 
Bio-grafía Escritos sobre La Biología y su Enseñanza.

Edición Extra-Ordinaria. ISSN 2027-1034 P.p 930 - 938

Memorias del VII Encuentro Nacional de Experiencias en la Enseñanza de la Biología y la Educación Ambiental y II Congreso Nacional de Investigación en la Enseñanza de la Biología

Investigación en Enseñanza de las Ciencias (CIEC). Revista EDUCyT, Vol. Extraordinario, Diciembre, 107-125. 